\title{
THE VALUE OF RADIUM IN NON-MALIGNANT GYNECOLOGICAL CONDITIONS. A LECTURE DELIVERED AT CHELSEA HOSPITAL FOR WOMEN.
}

BY KEITH VARTAN, F.R.C.S.ENG.

SINCE its introduction into therapeutics, radium has enjoyed a varying popularity, at first being treated with scepticism, and later heralded as a panacea for all ills, and it is only within the last few years, as a result of careful observation by a number of clinicians all over the world, that its correct value is becoming appreciated.

The non-malignant gynæcological conditions for which radium has the most value are : (I) Suppression of irregular uterine hæmorrhage ; (2) Fibroids ; (3) Certain cases of intrinsic dysmenorrhœa ; (4) Certain cases of endometriosis.

$\mathrm{Ra} \mathrm{Ba} \mathrm{Br}_{4}$ radium is used in the form of a salt, and from it are given off the $a, \beta$ and $\gamma$ rays, the first two of which are particulate emanations, the $a$ rays being helium nuclei, to which are attached one positively charged electron, and the $\beta$ rays being negatively charged electrons. The $\gamma$ rays are rays having the same velocity as light but having a much shorter wave-length. Now the shorter the wave the greater the penetration. For this reason, and also because the $a$ and $\beta$ rays are harmful to the tissues, these $a$ and $\beta$ rays are screened off, and are not used. For this purpose there is a choice of platinum, lead, silver and brass, in that order of efficacy, $0.5 \mathrm{~mm}$. of platinum being as efficacious as $2^{\circ} \circ \mathrm{mm}$. of brass. For utility therefore platinum is preferred. All these metals produce secondary rays which are screened from the tissues by rubber.

The therapeutic use of radium is based on two main facts : (I) That its effect varies inversely as the square of the distance, and its effect is therefore essentially local ; (2) That the more embryonic and more active the cell the more sensitive is it to radium.

That radium suppresses menstruation is known, but does it do so by acting on the endometrium or on the ovaries?

The supporters of the one school of thought allege that radium converts endometrium into connective tissue, and necrosis is caused by secondary rays improperly screened, which leads to healing and obliteration of the endometrial cavity. To support this they state that only 20 per cent. of women show menopausal symptoms, and also that in assuming the ovaries to have been affected the German school have failed to realize that the normal ovary is situated $3 \frac{1}{2}$ in. from the uterus, and to apply the law of the inverse square to this case.

The German school, however, believe the ovaries to be affected on the following grounds : (I) There is microscopic evidence of more or less complete destruction of the Graafian follicles, with fibrosis of the interstitial tissue and atrophy of the whole organ ; (2) The cells themselves show cloudy swelling, vacuolation, shrinking and ultimate replacement by fibrous tissue.

This theory is not without clinical support either, for it is not uncommon for a patient to have one or two menstrual periods before cessation, and sometimes, after a prolonged period of amenorrhœa, the menses may recommence. Now on the theory of fibrosis of the endometrial cavity, it would be possible to explain the gradual suppression of menstruation, but it is not possible in my opinion to explain its recommencement. 
If, however, one assumes the ovary to be affected then an explanation is forthcoming, as follows : Radium is given, and all actively growing follicles are destroyed. One or two, however, are on the point of rupturing and do so, causing one or two periods. After a prolonged period of amenorrhœa, follicles which previously were dormant, and not undergoing sufficiently active metabolism to be destroyed by radium,-mature and re-establish menstruation.

The balance of present-day opinion is in favour of the latter theory.

Before radium is given to suppress irregular hæmorrhage the full potential significance of the bleeding must be realized. In order to do this it is essential, firstly, to exclude the hæmorrhages associated with pregnancy, and hyperpiesis and then by physical examination to exclude carcinoma, submucous fibroids, polypi retained products, adenomata, and a variety of other conditions. If after these investigations nothing is found, one may assume that endocrine misbalance is the cause, and treat it accordingly, viz. : by suppressing the action of the ovary, subject to one very important consideration, namely, the age of the patient.

If the patient is below 40 then the menopausal symptoms, headache, flushings and sweatings are apt to be appallingly severe, and in some cases atrophy of the vulva and kraurosis occurs. Therefore the creation of a permanent and abrupt menopause below this age is undesirable. Between the ages of 40 and 43 it is well to be guarded in giving a prognosis of the after-effects. Above this age irradiation is indicated.

Both radium and X-ray should be considered and the following factors will help in deciding which to use.

(I) Radium is essentially more local in its action, therefore the effect on the ovaries will tend to be less, as will also the general physical disturbance.

(2) If the patient suffers from pure menorrhagia then the application of X-ray without other procedure may be justified and has the advantage of needing no anæsthetic, and occasioning no confinement to bed. If malignancy or other conditions cannot be excluded then an anæsthetic is given, the uterus explored, and at the same time a radium tube is inserted.

If possible it is better to wait a week between curetting and inserting the radium, for by curetting, the lymph spaces are opened up and severe reaction sometimes results.

Fifty mgm. of radium screened by $0^{\circ} 5 \mathrm{~mm}$. platinum and $2 \cdot 0 \mathrm{~mm}$. rubber are inserted, not into the cervix but into the fundus of the uterus, thus minimizing parametrial reaction and avoiding stricture of the cervix. The duration varies with different people, between twenty-four and seventy-two hours. The optimum time is probably forty-eight hours, and if the desired effect is not at first achieved, it can be repeated.

X-rays may be applied to create amenorrhœa at one application, or better, the administration can be spread over three or four months, and thus bring about a gradual change.

If the first method is used then a continuous current of 180 kilovolts filtered by $0.5 \mathrm{~mm}$. copper is applied by two to four ports of entry, usually one on either side anteriorly, one or two posteriorly. If the gradual method is used then an interrupted current of $I_{50}$ kilovolts is used filtered through $2 . \mathrm{mm}$. of aluminium. One port of entry is used on each of three successive days and the treatment is repeated at monthly intervals, three usually sufficing. 
The sequelæ are a prolonged and severe period, possibly a slight one and then amenorrhœea. This treatment has been elaborated by Dr. Finzi at St. Bartholomew's Hospital and is certain in competent hands.

The sequelæ of the radium therapy were worked out by Dr. Donaldson at St. Bartholomew's with a series of roo cases. Only is had no menopausal symptoms, $5^{\circ}$ put on weight, only 7 had dyspareunia, the last point suggesting that the criticism that radium scars the vagina is unjustified. The end-results of a series collected by Asherson at University College Hospital in a series of 63 cases : 55 were cured with one application, 6 required a second application, 2 untraced.

Asherson also has figures for cases in which radium was given to people below the age of 40 in order to produce a temporary menopause. Twenty-three cases treated : 16 restored to normal menstruation after a short period.

The difficulty in these cases is to be sure that a temporary, and not a permanent menopause is going to be caused. The dose in these cases was $50 \mathrm{mgm}$., and the time varied between eight and fifty hours. An observation arises out of this, namely, that the younger the patient the greater the dose necessary to produce amenorrhœea.

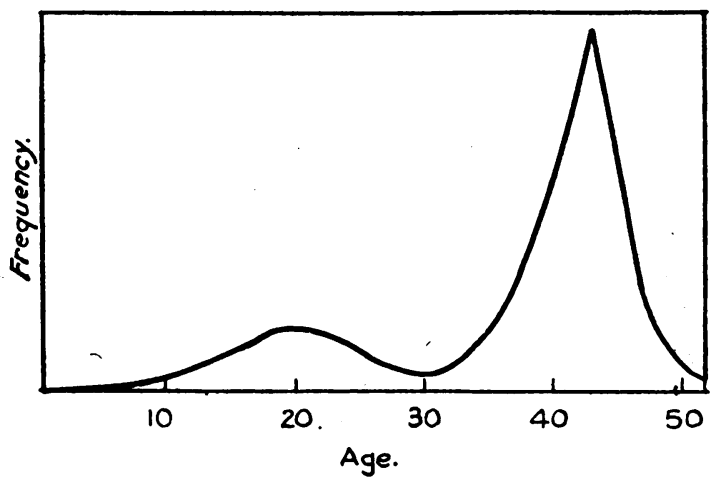

The foregoing remarks apply to irregular hæmorrhage, due either to essential menorrhagia or to the condition known for want of a better term as subinvolution, there is also, however, the condition of metropathia hæmorrhagica to be considered.

Now metropathia hæmorrhagica is a condition described by Dr. Wilfred Shaw, in which the uterus is larger than normal, the myometrium being hypertrophied and the endometrium polypoid. The glands are very numerous and are cystic and lined with columnar epithelium. The stroma is very cellular. On the endometrial surface are areas of hæmorrhagic necrosis, from which bleeding so severe as to cause profound: secondary anæmia may take place.

Accompanying these uterine changes there is always a cyst in one ovary, but no corpus luteum.

The age incidence is interesting and has an important bearing on the treatment.

From the diagram it is seen that the bulk of the cases occur at about 45 .

For these cases it is always worth while attempting to suppress hæmorrhage with radium.

A certain number of cases, however, occur in young girls of 20 to 25 , in which case the problem is different. In these cases a small dose of radium, viz.: $50 \mathrm{mgm}$. for four hours, has been given by Polak of New York, and in his cases normal menstruation has been established and some of his patients have subsequently married and borne children. 
It would seem to be worth while trying radium in these cases therefore, for the only satisfactory alternative is hysterectomy, in which case sterility is ensured.

Next we have to consider the position radium occupies in the treatment of fibroids.

Women over the age of 40 with small fibroids are no longer compelled to await the menopause, if not wishing to undergo a major surgical procedure, a process which is slow and incapacitating. Instead, by means of irradiation, suppression of menstruation can be made to occur and the patient be thus relieved.

As with everything else, however, there are rules which govern the use of radium in cases of fibroids :-

(I) The patient must be over 40 ;

(2) Hæmorrhage must be the cardinal symptom ;

(3) There must be no pressure symptoms ;

(4) The fibroids must not be pedunculated;

(5) There must be no sign of degeneration;

(6) No pelvic inflammation ;

and it will be seen by this list that the field is considerably limited.

Whether to use radium or X-rays is decided along the same lines as in the case of of suppressing uterine hæmorrhage, but it is well to remember that fibroids are associated with sterility and sterility with carcinoma corporis.

Now what are the objections to using radium?

Firstly, that although the hæmorrhage will be controlled and the fibroid shrink, yet it will not disappear, it may even grow again. Then it may degenerate, and in fact it is more likely to after radium, for the blood supply is interfered with. This explains why submucous ones should not be treated for in this case. Sloughing is almost certain to occur.

Again, malignant change may have been overlooked. It occurred in 6 per cent. in one series (Polak).

A large proportion of fibroids are accompanied by tubovarian disease in some form or another, this figure being placed as high as $5^{\circ}$ per cent. by some observers.

Also the inflammatory reaction, which radium always causes, will light up any latent adnexal inflammation which previously may have been entirely unsuspected.

Lastly, pressure symptoms are not relieved.

On the other hand, what advantages can radium offer over surgery?

Firstly, there is no mortality.

Secondly, should radium not be satisfactory surgery can still be fallen back upon.

Thirdly, it definitely is the treatment of choice when the patient is what is known as a poor operative risk. Suffering perhaps from carditis, chronic nephritis, or diabetes, uncontrolled by insulin.

In one series of cases, 206 patients were treated, 200 ceased menstruating and were relieved of symptoms. In the remaining 6 cases surgery had to be resorted to, and in each case it was because the case in the first place was unsuited to radium, submucous fibroids subseyuently being found on each occasion.

In $\mathrm{I} 30$ of these same cases the tumour shrunk to half its original size, and in 70 of them to all intents and purposes it had disappeared.

The last condition, for which radium has been, and is being satisfactorily used, is primary intrinsic dysmenorrhœa. This is a condition in which the dysmenorrhœa 
part of the clinical picture of a general hypoplasia of the genitalia. These are two main types: (1) The anteflexed, retroposited uterus with a small cervix; (2) a uterus with acute forward or backward flexion.

In these cases, when the standard and time-worn remedies have been tried, the cervix may be gradually dilated with Hegar's graduated sounds and a 50-mgm. radium tube is then inserted high up into the body of the uterus and is left there for four hours. Menstruation is not stopped but dysmenorrhœa is relieved.

Is it simply mechanical or psychological, or is it that the ovary has in some way been stimulated? A satisfactory explanation is not yet available, but if figures are required Polak's series could not be bettered : 36 cases ; 36 cures, 9 subsequently have become pregnant and 6 have live children.

In addition to these more well-established conditions for which radium $h$ is been used, there are one or two others, for instance, the diffuse adenomyoma such as occurs in the recto-vaginal septum, and which would be beyond the bounds of prudent surgery. Small doses have been used too in cases of amenorrhœa in the hope of stimulating the flagging ovary.

\section{Summary.}

Increasing experience has shown that the value of radium in non-malignant gynæcological conditions is less than was at one time thought. It is, however, of proven worth : (I) In suppression of irregular uterine hæmorrhage; (2) in the treatment of metropathia hæmorrhagica; (3) in the treatment of selected cases of intraumural fibromyomata; (4) in the treatment of otherwise intractible dysmenorrhœa.

\section{CASES DEMONSTRATED AT THE F.R.C.S. CLASS. A Case of Cervico-facial Swelling.}

A CHEERfuL, intelligent, healthy-looking little girl, aged I2, was shown to the F.R.C.S. candidates on March 20. Her home was in Streatham and her furthest voyage of exploration to date has been to her present hospital near Paddington. (The import of this statement will be obvious later.) She was suffering from a cervico-facial swelling, the history of which was as follows: In December, 1932, she had two teeth extracted from the left lower jaw, and a week later received a blow over this area of her face. Yet another week and a swelling began slowly to develop under the angle of the jaw on the left side, which has since steadily increased in size.

As presented, this swelling was some four inches in diameter, a dusky red colour all over, painless to palpate, attached firmly to the mandible and the sterno-mastoid, and felt definitely indurated. There was limitation of movement in the temporo-mandibular joint, but not of the tongue, the inside of the mouth being free of blemish, though the mass was easily palpated through the buccal mucous membrane. On the outer surface of the lump were three small incisions, from which small quantities of yellowish serous fluid were oozing. No glands were palpable.

The diagnosis of actinomycosis was made by the majority of candidates, though anthrax, lupus, and even cellulitis did occur. It is not so much the diagnosis itself one would like to discuss as the method by which it was arrived at. I think it is a fair statement to say that actinomycosis, though perhaps not rare, is sufficiently uncommon to make an initial diagnosis somewhat of a query. And yet only two candidates asked for an X-ray of the mandible (which incidentally showed no bony changes whatsoever) 